\title{
Encoded latent membrane protein 1 of Epstein-Barr virus on follicular dendritic cells in residual germinal centres in Hodgkin's disease
}

\author{
J van Gorp, K C Jacobse, R Broekhuizen, J Alers, J G van den Tweel, R A de Weger
}

\begin{abstract}
Aims-To determine if there is an association between Epstein-Barr virus (EBV) infection and Hodgkin's disease.

Methods-Fifty cases of Hodgkin's disease and 25 reactive lymph nodes were screened for the presence of EBV-RNA (EBER) using in situ hybridisation, and for the expression of EBV encoded latent membrane protein 1 (LMP-1) by immunohistochemistry. Results-In $42 \%$ of the cases of Hodgkin's disease, EBER was detected in the nuclei of the malignant cells, and in LMP-1 expression was found $36 \%$. Both EBER and LMP-1 positivity were seen in $34 \%$ of the cases. An additional finding was the presence of LMP-1 on follicular dendritic cells in residual germinal centres in two cases of Hodgkin's disease. EBER was not detected in these germinal centres. In reactive lymph nodes only occasional EBER positive, small, lymphoid cells were found, without LMP-1 expression.
\end{abstract}

Conclusions-These results show a strong correlation between the presence of EBER and the LMP-1 expression in the Reed-Sternberg cells. They corroborate a role for $E B V$ in at least some cases of Hodgkin's disease. LMP-1 is probably presented as an immune complex in the germinal centres, as part of an immune response against EBV.

(F Clin Pathol 1994;47:29-32)

There is increasing evidence for an association between Hodgkin's disease and EpsteinBarr virus infection (EBV). EBV-DNA has been detected in specimens of Hodgkins disease using Southern blotting. ${ }^{1}$ The EBV genomes present in these cases seemed to be of monoclonal origin, suggesting infection early in development of the disease. Using a very sensitive EBV-RNA in situ hybridisation technique, EBV-RNA (EBER) was found in Reed-Sternberg cells and its variants in nearly $50 \%$ of the cases. ${ }^{23}$ Furthermore, in $39-48 \%$ of the cases of Hodgkin's disease, EBV encoded latent membrane protein 1 (LMP-1) expression can be detected only in ReedSternberg cells and their variants. ${ }^{34} \mathrm{LMP}-1$ is thought to have a role in EBV induced cell transformation ${ }^{5}$ and is expressed in several EBV associated malignancies. Little is known about a specific immune response to the EBV positive malignant cells in Hodgkins disease.

In this study we screened 50 specimens of Hodgkin's disease for the presence of EBVRNA (EBER) and for the expression of LMP, to determine the correlation of both EBV gene products. Special emphasis was given to EBV infection in the pre-existing lymph node component

\section{Methods}

Formaldehyde fixed, paraffin wax embedded lymph nodes from 50 patients with Hodgkin's disease and 25 reactive lymph nodes were studied. In two cases of Hodgkin's disease snap frozen material, stored at $-80^{\circ} \mathrm{C}$, was also studied. Specimens of Hodgkin's disease comprised one case of lymphocyte predominant Hodgkin's disease (LPHD), 32 cases of nodular sclerosing (NSHD), 16 cases of mixed cellularity type (MCHD), and one case of lymphocyte depleted (LDHD), according to the Rye classification. The nonmalignant lymph nodes displayed histologically non-specific reactive features with either predominantly follicular or interfollicular hyperplasia.

For in situ hybridisation a fluorescein conjugated DNA-oligonucleotide mixture of two 30-mers was used, complementary to the two nuclear EBER-RNAs encoded by EBV (Dakopatts (Dako), Glostrup, Denmark). RNA in situ hybridisation was performed on $6 \mu \mathrm{m}$ tissue sections, placed on organosilane pretreated glass slides. After dewaxing and rehydration the sections were pretreated with proteinase $\mathrm{K}(3 \mu \mathrm{g} / \mathrm{ml}$ in phosphate buffered saline (PBS) for 30 minutes at $37^{\circ} \mathrm{C}$. The slides were dehydrated again. One to two drops $(15-30 \mu \mathrm{m})$ of EBV (EBER) oligonucleotides/fluorescein isothiocyanate (FITC) (undiluted; Dako) were added and covered with a coverslip for two hours at $37^{\circ} \mathrm{C}$. The coverslip was removed and the sections were immersed in PBS. After preincubation with $10 \%$ normal swine serum a three-step peroxidase reaction was applied: the first step included mouse anti-FITC (Dako). The second, rabbit anti-mouse immunoglobulin, and the third, swine anti-rabbit immunoglobulin, both conjugated to horseradish peroxidase (both from Dako). Sections were counterstained with haematoxylin.

Immunohistochemical staining was performed on $6 \mu \mathrm{m}$ tissue sections that were placed on organosilane pretreated glass slides. After dewaxing, endogenous peroxidase activity was blocked by incubating the slides in
Correspondence

Accepted for publication 29 July 1993 
$1.5 \% \mathrm{H}_{2} \mathrm{O}_{2}$ in methanol for 30 minutes. The LMP-1 antibody cocktail (CS.1-4) was kindly provided by Professor AB Rickinson, University of Birmingham. After preincubation with $10 \%$ normal horse serum for 10 minutes a three-step immunoperoxidase procedure was applied: the first step included the LMP-1 antibodies (CS.1-4); the second, biotinylated horse anti-mouse immunoglobulin (Vector, Burlingame, California, USA); and the third, streptavidin peroxidase (Boehringer, Mannheim, Germany). The peroxidase activity was developed by 3,3 diaminobenzidine tetrahydrochloride using nickel ammonium sulphate-6-hydrate and $\mathrm{H}_{2} \mathrm{O}_{2}$ as substrate. The sections were counterstained with nuclear fast red or haematoxylin. For the negative controls, LMP-1 antibody was omitted.

Additional immunohistochemical staining was performed on serial sections of snap frozen material stored at $-80^{\circ} \mathrm{C}$ of two cases of MCHD. Sections $(6 \mu \mathrm{m})$ were placed on organosilane coated glass slides. Sections were fixed in acetone for 10 minutes. Endogenous peroxidase activity was not blocked. The antibodies LMP-1 (CS1-4) and KiM4 (Behringwerke, Marburg, Germany), an antibody with specific reactivity to follicular dendritic cells were used. A three-step immunoperoxidase procedure was performed with nickel enhancement, as described above.

\section{Results}

EBV-RNA (EBER) was found in $21(42 \%)$ cases of Hodgkin's disease and was restricted to the nuclei of Reed Sternberg cells and their variants. In MCHD $13(81 \%)$ cases contained EBER; only eight (25\%) cases of NSHD were positive. In contrast to LMP-1, several cases of both MCHD and NSHD showed positivity for EBER in a few scattered small lymphocytes. In some of these cases the malignant cells were negative. In four reactive lymph nodes occasional EBER positive small lymphoid cells were also detected.

LMP-1 expression was found in 18 of the $50(36 \%)$ cases of Hodgkin's disease, 12 being MCHD and six NSHD (table). Expression was restricted to Reed-Sternberg cells and their variants, and was mostly seen on the cell membrane and in the Golgi region. No LMP-1 expression was found in the small reactive lymphoid cells in Hodgkin's disease, nor in any of the 25 reactive lymph nodes. In $17(34 \%)$ cases of Hodgkin's disease both EBER and LMP-1 were detected.

In two cases of MCHD LMP-1 expression was found in some, but not all, preserved germinal centres (fig 1). No LMP-1 expression was found in pre-existing germinal centres in any of the other cases of Hodgkin's disease, nor in any of the reactive lymph nodes. Distribution in the germinal centres in the two cases of Hodgkin's disease was restricted to the plasma membrane of the follicular dendritc cells. This restriction in the germinal centres was confirmed by serial sections on snap frozen sections using LMP-1 and KiM4 (fig 2). All germinal centres were positive in the frozen sections in these two cases.

No positivity for EBER was detected in the germinal centres. In both cases the Reed Sternberg cells and variants were positive for EBER and LMP-1.

\section{Discussion}

EBV was present in $42 \%$ of the patients with Hodgkin's disease. EBV-RNA was predomi-
Figure 1 LMP-1 in a preserved germinal centre. Distribution suggests staining of the follicular dendritic cells. $A$ LMP-1-positive Reed-Sternberg cell is present just outside the lymph follicle.

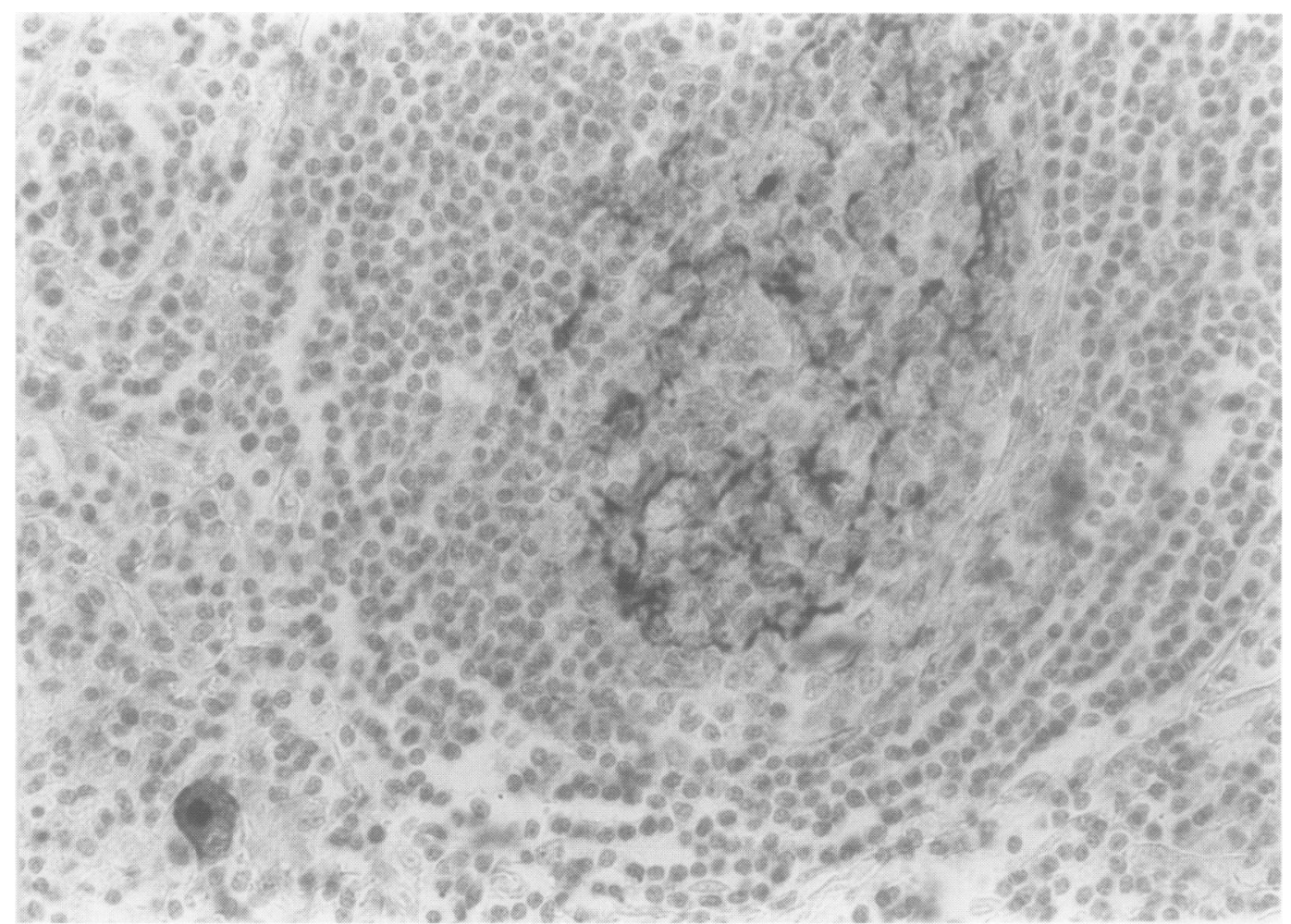


Results of EBER in situ hybridisation and LMP-1 expression among 50 cases of Hodgkin's disease studied

\begin{tabular}{lcccc}
\hline & $n=$ & $L M P+$ & $E B E R+$ & $L M P+/ E B E R+$ \\
\hline Hodgkin's disease & 50 & $18(36 \%)$ & $21(42 \%)$ & $17(34 \%)$ \\
Lymphocyte predominant & 1 & 0 & 0 & 0 \\
Nodular sclerosing & 32 & $6(19 \%)$ & $8(25 \%)$ & $5(16 \%)$ \\
Mixed cellularity & 16 & $12(75 \%)$ & $13(81 \%)$ & $12(75 \%)$ \\
Lymphocyte depleted & 1 & 0 & 0 & 0 \\
\hline
\end{tabular}

nantly detected in malignant Reed-Sternberg cells. Furthermore, in most of the EBV positive cases of Hodgkin's disease, Reed-Sternberg cells expressed the EBV protein LMP-1. We found a strong correlation between LMP-1 expression and EBER positivity in Reed-Sternberg cells and variants. As EBER positivity in small $B$ cells can be detected in healthy people with EBV infec-
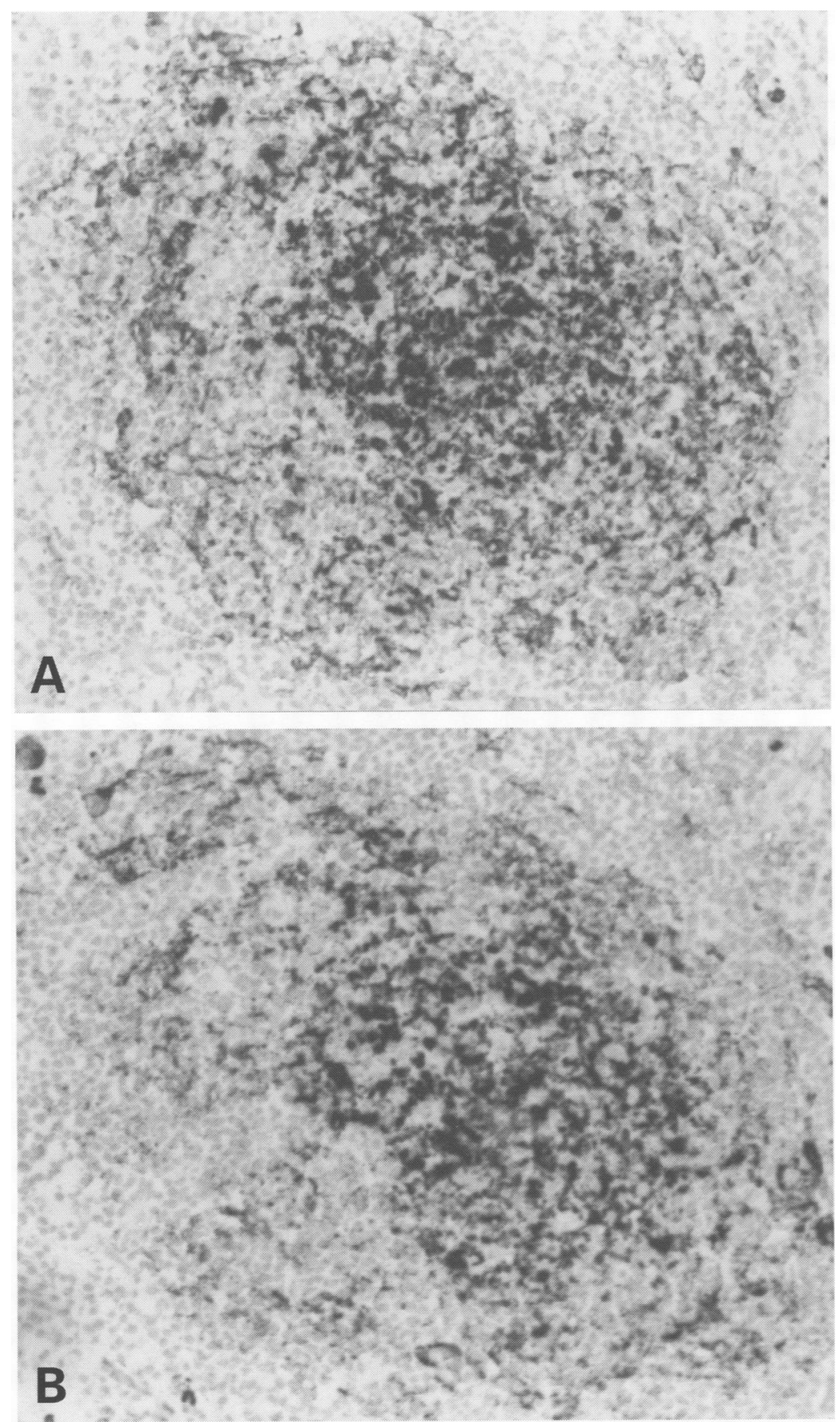

Figure 2 Serial sections of frozen material: $(A)$ immunoreactivity with KiM4; (B) with LMP-1. tion, it probably does not contribute to Hodgkin's disease. Lack of LMP-1 expression in these cells supports this idea. These findings are consistent with those of other studies $^{3}$ and suggest a pathogenetic role for EBV in Hodgkin's disease. MCHD (75\%) showed a stronger association with EBV than NSHD (25\%).

The detection of LMP-1 expression in follicular dendritic cells in the germinal centres of two cases of Hodgkin's disease has not been described before. There are two plausible explanations for this phenomenon. First, LMP-1 expression on follicular dendritic cells may be a reflection of the presence of EBV in these cells. Follicular dendritic cells show strong expression of CD21. The monoclonal antibodies clustered in CD21 include antibodies against the $\mathrm{C} 3 \mathrm{~d}$ receptor (CR2) present on B cells. EBV infection of $B$ cells follows after specific binding to this receptor. Some of the monoclonal antibodies against CD21 (HB5 and anti-B2) have been found to react with related, but not identical, antigens, expressed on epithelium of the uterine cervix and oropharynx. ${ }^{67}$ As EBV can regularly be detected in these epithelia, this surface molecule on epithelial cells may serve as an EBV receptor. The association of the virus with Burkitt's lymphoma, with B cell lymphoproliferative disorders, and hairy cell leukcoplakia in immune deficient patients, and with nasopharyngeal carcinoma concurs with this theory. CD21 expression has been found on Reed-Sternberg cells and variants containing EBV-DNA. ${ }^{8}$ If the follicular dendritic cells in the two cases of MCHD were infected by EBV a positive EBER in situ hybridisation signal would be expected, but we were not able to detect EBER positivity in these germinal centres in serial sections. Therefore, infection of follicular dendritic cells by EBV is not very likely.

The second, more plausible, explanation of LMP-1 expression on follicular dendritic cells is that the latter are believed to play an important part in the retention of antigen in lymphoid follicles and in the generation of B memory cells by binding of antigen-antibody complexes and presentation of these complexes to the follicular B cells. As the Reed-Sternberg cells and variants strongly expressed LMP-1 in these two cases of MCHD, it is conceivable that the follicular dendritic cells were binding the LMP protein in germinal centres in the form of an immune complex. This might have been an antibody response to EBV, directed against the LMP1-antigen.

The malignant cells in Hodgkin's disease are believed to express only part of the EBV latent proteins-EBNA-1 and LMP-1-and probably LMP-2. ${ }^{9}$ Antibody cocktail CS1-4 is directed against LMP-1 and does not react with LMP-2a or LMP-2b. The expression of LMP-2 on Reed-Sternberg cells has not yet been demonstrated by immunohistochemistry. Although foreign protein is expressed on relatively few malignant cells (Reed-Sternberg cells and variants) in a 
prominent number of cases of Hodgkin's disease, the immune system does not seem to be able to deal with these cells, despite the huge reactive lymphoid infiltrate surrounding these cells. Cytotoxic $\mathrm{T}$ lymphocytes are the most important defense system against viral infected cells, but EBNA-1 does not seem to be a suitable target antigen for cytotoxic lymphocytes. ${ }^{10}$ The cytotoxic lymphocytes response against LMP-1 is more complicated and does not seem to be present in everyone. ${ }^{11}$ LMP-2 probably is the most important target for cytotoxic lymphocytes but patients with Hodgkin's disease are known to have an impaired immune surveillance. Whether antibody response to LMP-1 contributes to the defense against EBV positive cases of Hodgkin's disease remains to be investigated.

C C Jacobse received a grant from Stichting Cacharyne 101, University Hospital, Utrect, The Netherlands.

1 Weiss LM, Strickler JG, Warnke RA, Purtilo DT, Sklar J. Epstein-Barr viral DNA in tissues of Hodgkin's disease. Am F Pathol 1987;129:86-91.
2 Wu TC, Mann RB, Charache $\mathrm{P}$, et al. Detection of EBV gene expression in Reed-Sternberg cells of Hodgkin's disease. Int $\mathcal{F}$ Cancer 1990;46:801-4.

3 Herbst H, Steinbrecher E, Niedobitek G, et al. Distribution of Epstein-Barr virus-harboring cells in Hodgkin's disease. Blood 1992;80:484-91.

4 Pallesen G, Hamilton-Dutoit SJ, Rowe M, Young LS. Expression of Epstein-Barr virus latent gene products in tumour cells of Hodgkin's disease. Lancet 1991;337: 320-2.

5 Wang D, Leibowitz D, Kieff E. An EBV membrane protein expressed in immortalized lymphocytes transformed established rodent cells. Cell 1985;43:831-40.

6 Young LS, Sixbey JW, Clark D, Rickinson AB. EpsteinBarr virus receptor on human pharyngeal epithelia. Lancet 1986;i:240-2.

7 Young LS, Dawson CW, Brown KW, Rickinson AB. Identification of a human epithelial cell surface protein sharing an epitope with the $\mathrm{C} 3 \mathrm{~d} /$ /Epstein-Barr virus receptor molecule of $\mathrm{B}$ lymphocytes. Int $\mathcal{f}$ Cancer 1989;43:786-94.

8 Jiwa NM, Van der Valk P, Mullink H, et al. Epstein-Barr virus DNA in Reed-Sternberg cells of Hodgkin's disease is frequently associated with CR2 (EBV receptor) is frequently associated with CR2 (EB

9 Kerr BM, Lear AL, Rowe M, et al. Three transcriptionally distinct forms of Epstein-Barr virus latency in somatic cell hybrids: cell phenotype dependence of virus promoter usage. Virology 1992;187:189-201.

10 Rickinson AB, Murray RJ, Brooks J, Griffith H, Moss DJ, Masucci MG. T cell recognition of Epstein-Barr virus associated lymphomas. Cancer Surveys 1992;13:53-79.

11 Murray RJ, Kurilla MG, Brooks JM, et al. Identification of target antigens for the human cytotoxic $T$ cell response to Epstein-Barr virus (EBV): implications for the immune control of EBV-positive malignancies. F Exp Med 1992;176:157-68. 